\title{
Esophagography in Patients With Esophageal Achalasia Diagnosed With High-resolution Esophageal Manometry
}

\author{
Takahisa Yamasaki, Toshihiko Tomita, ${ }^{*}$ Sumire Mori, Mayu Takimoto, Akio Tamura, Ken Hara, Takashi Kondo, Tomoaki Kono, \\ Katsuyuki Tozawa, Yoshio Ohda, Tadayuki Oshima, Hirokazu Fukui, Jiro Watari, and Hiroto Miwa
}

Division of Gastroenterology, Department of Internal Medicine, Hyogo College of Medicine, Nishinomiya, Hyogo, Japan

\begin{abstract}
Background/Aims
High-resolution esophageal manometry (HREM) is considered to be the gold standard for the diagnosis of achalasia. However, the Japan Esophageal Society recommends that esophagography is also accurate in either diagnosing or excluding the disorder. Accordingly, we compared the efficacy of esophagography and HREM in diagnosing achalasia patients with upper gastrointestinal symptoms.

\section{Methods}

HREM was performed in 126 patients with dysphagia. The final diagnosis of achalasia was done using HREM. Demographic data, symptoms, quality of life (QOL) were also obtained. We assessed the patients who were not able to be diagnosed by esophagography and compared the diagnostic values for esophagography with HREM-based achalasia diagnosis as the gold standard.
\end{abstract}

\section{Results}

A total of 48 cases of patients with achalasia, including 21 men and 27 women (mean age, $48.4 \pm 19.6$ years), were included in the study. Two patients were excluded. Of the remaining 46 patients, $36(78.3 \%)$ patients were diagnosed as having achalasia by esophagography. The diagnostic sensitivity, specificity, and accuracy of esophagography were $78.3 \%, 88.0 \%$, and $83.0 \%$, respectively. Patients with type III achalasia had significantly lower physical QOL score than those with type I or II achalasia. Although the mental QOL score in patients with type III achalasia tended to decrease compared with that in patients with type I and II achalasia, the difference was not statistically significant.

\section{Conclusions}

Diagnosing esophageal achalasia by using esophagography alone has limited yield. Therefore, HREM should be used in patients with dysphagia and in whom achalasia cannot be diagnosed using EGD or esophagography.

(J Neurogastroenterol Motil 2018;24:403-409)

Key Words

Esophageal achalasia; Esophagography; High-resolution esophageal manometry

Received: December 16, 2017 Revised: March 27, 2018 Accepted: May 21, 2018

() This is an Open Access article distributed under the terms of the Creative Commons Attribution Non-Commercial License (http://creativecommons. org/licenses/by-nc/4.0) which permits unrestricted non-commercial use, distribution, and reproduction in any medium, provided the original work is properly cited.

*Correspondence: Toshihiko Tomita, MD, PhD

Division of Gastroenterology, Department of Internal Medicine, Hyogo College of Medicine, 1-1, Mukogawa-cho, Nishinomiya, Hyogo 663-8501, Japan

Tel: +81-798-45-6662, Fax: +81-798-45-6661, E-mail: tomita@hyo-med.ac.jp 


\section{Introduction}

Esophageal achalasia is a rare disease with a prevalence of 1.08 cases per 100000 persons, and most frequently occurs in younger patients aged 20-40 years. ${ }^{1}$ The Japan Esophageal Society revised its guidelines in 2012, for the first time in approximately 30 years, to address patients with esophageal achalasia. They recommend that esophagography be performed as a diagnostic method. ${ }^{2}$ The society also recommend that physicians classify such patients based on the type and degree of esophageal dilatation, as well as the specific characteristics of esophageal radiographic findings.

However, currently, esophagography is not performed regularly in clinical practice in the United States and Europe, and also differs from the current standard diagnostic procedures followed in Japan.

High-resolution esophageal manometry (HREM) with multichannel pressure sensors is a recently developed technology allowing for a dynamic and comprehensive assessment of esophageal movement in real time. A novel classification based on HREM findings has also been proposed. ${ }^{3}$

In this study, we compared the efficacy of esophagography and HREM in diagnosing achalasia patients with upper gastrointestinal symptoms based on the Chicago classification. In addition, we assessed the association between the patients' health-related quality of life (QOL) and type of achalasia.

\section{Materials and Methods}

\section{Study Design}

HREM was performed in 126 men and women with dysphagia, aged 18-80 years, who visited our hospital between June 2012 and July 2015. Patients with organic disease, as detected with esophagogastroduodenoscopy (EGD), were excluded from this study. The other exclusion criteria were as follows: (1) diseases that cause epigastric digestive symptoms, such as malignant tumors, peptic ulcers, heart disease, or systemic diseases (eg, neurological conditions including Parkinson's disease or metabolic diseases including diabetes); (2) clear epigastric digestive symptoms due to excessive drinking, overeating, use of non-steroidal anti-inflammatory drugs, or excessive stress; (3) a history of surgery in the upper gastrointestinal tract, including the stomach or esophagus; (4) severe liver or renal dysfunction; (5) presence or suspicion of a disease complicated by a psychiatric disorder; (6) pregnancy or lactation, and intention to become pregnant during the study period among women; and (7) unsuitability for participation in this study based on the judgment of the attending physician. The diagnosis of esophageal achalasia was based on the Chicago classification (version 2). Lastly, we examined patients with esophageal achalasia having Eckhardt scores of 3 or higher. This study was approved by the ethics committee of Hyogo College of Medicine (Approval No. 1844), and written informed consent was obtained from all participants.

\section{Symptom and Quality of Life Assessment}

Symptoms of dysphagia, chest pain, and regurgitation were assessed using a self-reported questionnaire, with the cumulative score used as a descriptor of difficulty in eating, pain with swallowing, and food impaction. We also evaluated QOL by using the SF-8 questionnaire. We further analyzed the summaries of physical QOL (physical component summary [PCS]) and emotional QOL (mental component summary [MCS]).

\section{Analysis of Esophagography}

A $100 \mathrm{~mL}$ volume of a $100 \%$ weight/volume barium sulfate suspension was administered orally to the patients in the standing position. Radiographs in anteroposterior and right anterior oblique projections were taken 1 minute after the administration without using an antispasmodic agent. The diagnosis of achalasia was based on findings indicative of a dilatated or tortuous esophagus, presence of food residue, barium retention in the esophagus, smooth-tapered appearance (bird beak sign) of the esophagogastric junction (EGJ), loss of or decrease in gastric air bubble, and abnormal esophageal motility. Achalasia was classified as straight, sigmoid, or advanced sigmoid type based on the type and degree of dilatation observed. Straight-type achalasia was diagnosed when the lines obtained along with the long axes of the lower esophagus crossed at an angle $(\alpha)$ of $\geq 135^{\circ}$. Sigmoid-type achalasia was diagnosed when the angle was $90^{\circ}-135^{\circ}$, and advanced sigmoid-type achalasia was diagnosed when the angle was $<90^{\circ}$. Images from barium esophagography were examined by 2 investigators, which led to the final diagnosis.

\section{High-resolution Esophageal Manometry}

HREM was performed using a solid-state system (INSIGHT; Sandhill Scientific, Highlands Ranch, CO, USA) with 36 circumferential pressure sensors spaced at $1 \mathrm{~cm}$ intervals and with a diameter of $5.3 \mathrm{~mm}$. The procedure was performed according to a wellaccepted standard protocol. The patients were required to fast after midnight on the night before the test. After calibration at $0 \mathrm{mmHg}$ and $100 \mathrm{mmHg}$ using externally applied pressure, the manometry assembly was placed transnasally and positioned in the stomach, 


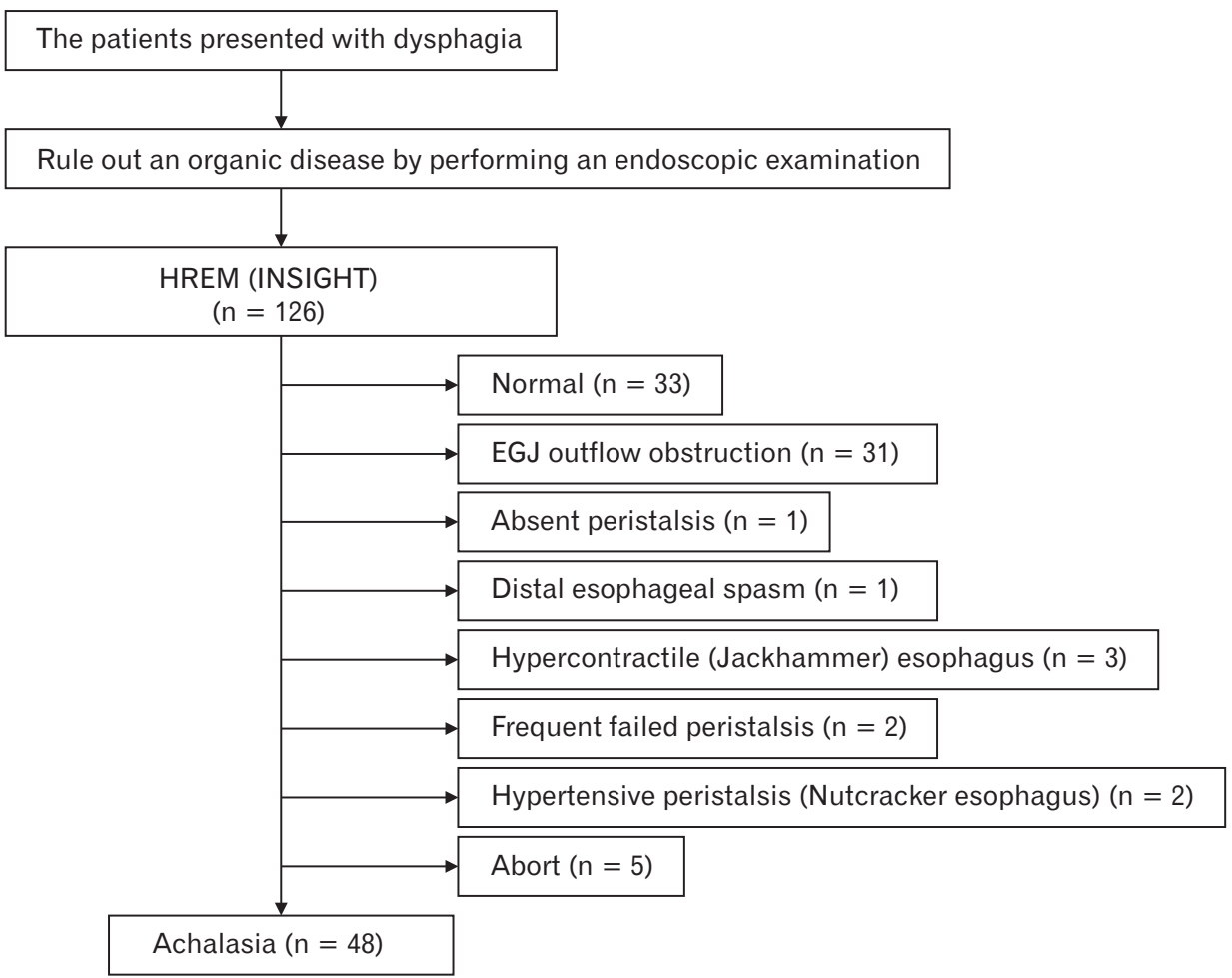

Figure 1. Subjects included in this study (study duration: June 2012-July 2015). HREM, high-resolution esophageal manometry; EGJ, esophagogastric junction. providing pressure measurements from the hypopharynx to the stomach with approximately 3 intragastric sensors. Thereafter, the patients were placed in the supine position and were asked to swallow $5 \mathrm{~mL}$ of water at room temperature 10 times at an interval of 20-30 seconds.

\section{Statistical Methods}

HREM plots were evaluated to assess esophageal motor function during each wet swallow according to the Chicago classification version $2 .^{3}$ This included the integrated relaxation pressure, distal contractile integral, distal latency, peristaltic breaks, and contractile front velocity. The diagnoses of esophageal motility disorders were grouped into 10 categories; normal motility, achalasia, EGJ outflow obstruction, absent peristalsis, distal esophageal spasm, hypercontractile (jackhammer) esophagus, weak peristalsis, frequent failed peristalsis, hypertensive peristalsis (nutcracker esophagus), and rapid contraction. The final diagnosis of esophageal achalasia was done using HREM.
Table 1. Demographic Characteristics of Achalasia Patients Diagnosed With High-resolution Esophageal Manometry

\begin{tabular}{lccc}
\hline & Type I & Type II & Type III \\
\hline Number of & $10(20.8)$ & $34(70.8)$ & $4(8.3)$ \\
patients (\%) & & & \\
Age (yr) & $48.3 \pm 22.8$ & $46.6 \pm 18.2$ & $63.8 \pm 21.3$ \\
Sex (M/F, n) & $6 / 4$ & $13 / 21$ & $2 / 2$ \\
IRP (mmHg) & $28.0 \pm 15.9$ & $38.4 \pm 16.0$ & $47.8 \pm 31.5$ \\
Symptoms (n) & & & \\
Dyspphagia & 7 & 23 & 2 \\
Reflux & 3 & 4 & 1 \\
Weight loss & 3 & 8 & 1 \\
Chest pain & 1 & 5 & 3 \\
\hline
\end{tabular}

$\mathrm{M}$, male; F, female; IRP, integrated relaxation pressure.

\section{Results}

\section{Enrollment Characteristics of the Patients}

On the basis of the Chicago classification (version 2), patients who visited our hospital with the chief complaint of dysphagia and underwent HREM ( $\mathrm{n}=126)$ were diagnosed as having a normal esophagus ( $\mathrm{n}=33$ ), EGJ outflow obstruction $(\mathrm{n}=31)$, absent peristalsis $(n=1)$, distal esophageal spasm $(n=1)$, hypercontrac- 

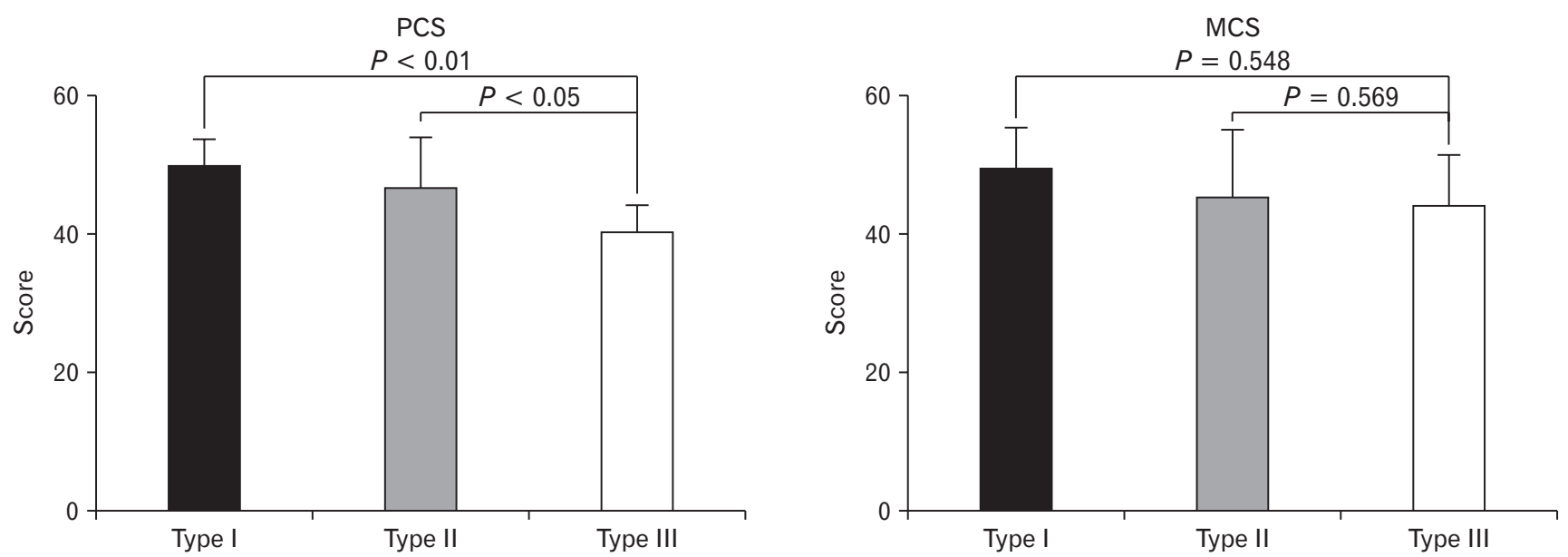

Figure 2. Comparison of quality of life according to achalasia type. PCS, physical component summary; MCS, mental component summary.

Table 2. Esophagography Findings of 36 Cases Diagnosed as Achalasia With High-resolution Esophageal Manometry

\begin{tabular}{lc}
\hline & $\mathrm{n}(\%)$ \\
\hline Dilatation type & $31(86.1)$ \\
Straight $\left(\mathrm{a} \geq 135^{\circ}\right)$ & $4(11.1)$ \\
Sigmoid $\left(90^{\circ} \leq \mathrm{a} \leq 135^{\circ}\right)$ & $1(2.8)$ \\
Advanced sigmoid $\left(\mathrm{a}<90^{\circ}\right)$ & $15(41.7)$ \\
Degree of dilation & $19(52.8)$ \\
I $(\mathrm{d}<3.5 \mathrm{~cm})$ & $2(5.5)$ \\
II $(3.5 \mathrm{~cm} \leq \mathrm{d}<6.0 \mathrm{~cm})$ & \\
III $(\mathrm{d} \geq 6.0 \mathrm{~cm})$
\end{tabular}

tile (jackhammer) esophagus $(\mathrm{n}=1)$, frequent failed peristalsis $(\mathrm{n}$ $=2)$, and hypertensive peristalsis (nutcracker esophagus) $(n=2)$. Five patients had difficulties during catheter insertion (Fig. 1). Altogether, 48 patients, including 21 men and 27 women (mean age, $48.4 \pm 19.6$ years), were diagnosed as having esophageal achalasia and included in the study. Ten $(20.8 \%)$ patients had type I achalasia, $34(70.8 \%)$ had type II, and $4(8.3 \%)$ patients had type III achalasia based on HREM findings. Table 1 provides the characteristics of the patients.

\section{Health-related Quality of Life}

Patients with type III achalasia had significantly lower PCS than those with type I or II achalasia. Specifically, the PCS score was $49.8 \pm 3.6,46.6 \pm 7.1$, and $40.3 \pm 3.8$ in patients with type I, II, and III achalasia $(P<0.05$ when comparing type II and type III, $P<0.01$ when comparing type I and type III). Although the MCS score in patients with type III achalasia tended to decrease
Table 3. Ten Cases Who Were Not Able to Be Diagnosed by Esophagography

\begin{tabular}{ccll}
\hline Case No. & Age (yr) & HREM & Esophagography \\
\hline 1 & 64 & Type I & Cork-screw \\
2 & 75 & Type II & Normal \\
3 & 76 & Type II & Cork-screw \\
4 & 79 & Type II & Normal \\
5 & 42 & Type II & Normal \\
6 & 81 & Type II & Cork-screw \\
7 & 25 & Type II & Normal \\
8 & 67 & Type III & Cork-screw \\
9 & 89 & Type III & Cork-screw \\
10 & 62 & Type III & Cork-screw \\
\hline
\end{tabular}

HREM, high-resolution esophageal manometry.

compared with that in patients with type I and II achalasia, the difference was not statistically significant (Fig. 2).

\section{Diagnosability of Esophagography}

A total of 46 patients underwent esophagography, and 36 (78.3\%) patients were diagnosed as having achalasia (Table 2). Of the remaining $10(21.7 \%)$ patients, $8(80.0 \%)$ were diagnosed as having a normal esophagus by EGD, without abnormal findings suggesting esophageal dilatation.

The breakdown of the 10 patients in whom a diagnosis of achalasia was not reached by using esophagography was as follows: 4 patients who exhibited a normal esophagus and 6 patients who presented with a corkscrew-like appearance in the esophagus and no bird beak sign in the lower esophageal sphincter (LES). All 

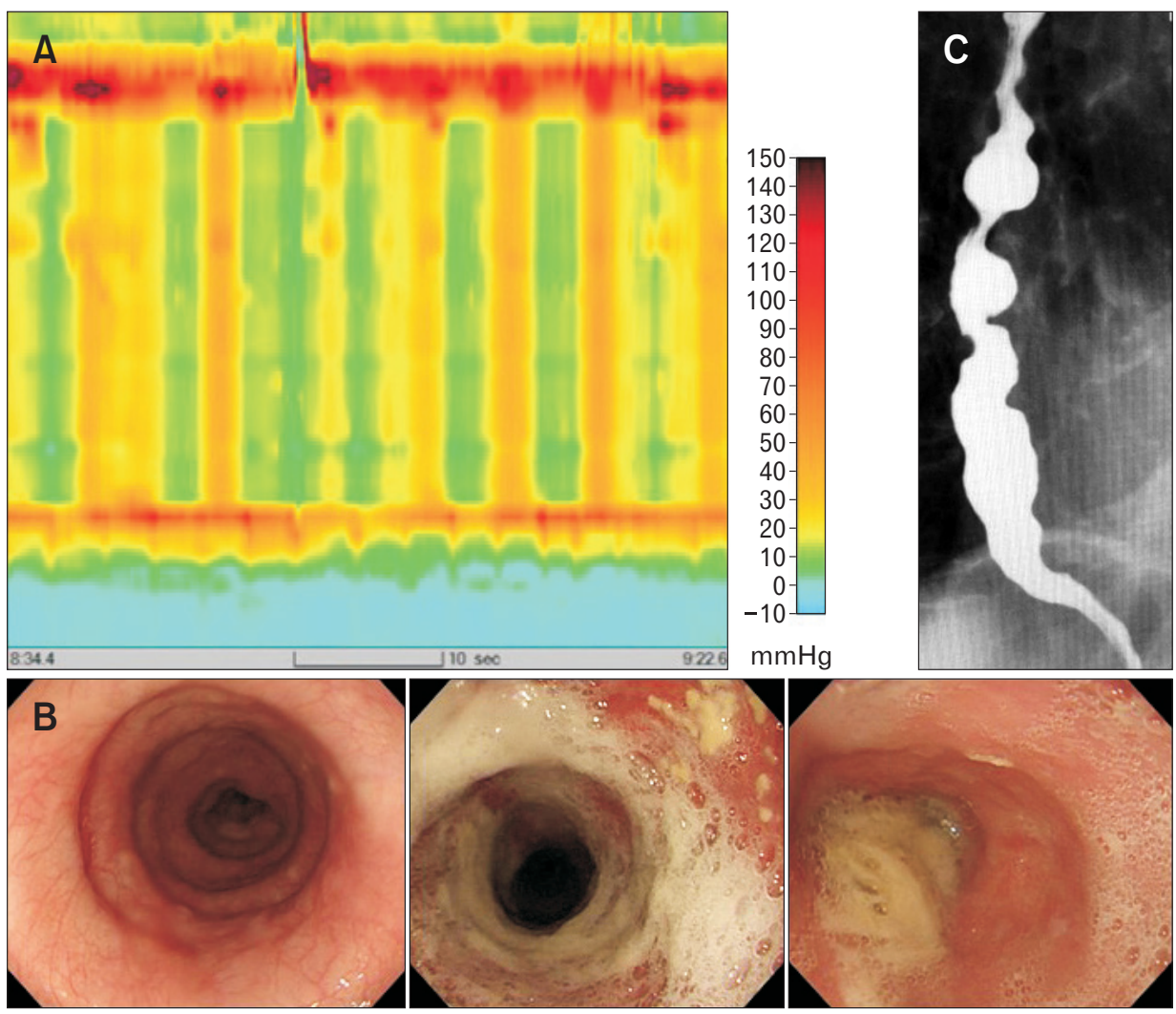

Figure 3. A case in which esophagography did not lead to a diagnosis of achalasia. (A) High-resolution esophageal manometry showed type II achalasia (integrated relaxation pressure $=44$ $\mathrm{mmHg}$ ). (B) Esophagogastroduodenoscopy showed spasticity and large amounts of food residue in the esophagus. (C) Esophagography showed spastic contraction from the middle thoracic esophagus to the esophagogastric junction.
Table 4. Diagnostic Accuracy by the Definition of Esophagography

\begin{tabular}{lccccc}
\hline & Sensitivity & Specificity & Accuracy & PPV & NPV \\
\hline Esophagography & 0.78 & 0.88 & 0.83 & 0.86 & 0.81
\end{tabular}

$\mathrm{PPV}$, positive predictive value; NPV, negative predictive value.

HREM findings in the 4 patients diagnosed as having a normal esophagus indicated type II achalasia. The HREM findings of the other 6 patients who showed a corkscrew-like appearance indicated type I ( $\mathrm{n}=1)$, II $(\mathrm{n}=2)$, and III $(\mathrm{n}=3)$ achalasia, with half of the patients presenting with type III achalasia (Table 3). Figure 3 shows a case in which esophagography did not lead to achalasia diagnosis.

Table 4 shows the diagnostic values for esophagography with HREM-based achalasia diagnosis as the gold standard. The diagnostic sensitivity, specificity, and accuracy of esophagography were $78.3 \%, 88.0 \%$, and $83.0 \%$, respectively.

\section{Discussion}

In this study, we compared the diagnostic efficacy of HREM and esophagography in patients who visited the hospital with dysphagia as the chief complaint. We found that the use of esophagography alone, which is commonly used to diagnose patients with esophageal achalasia in Japan, had limitations.

Upper endoscopy, esophagography, esophageal manometry, and histopathological examination of the esophageal muscle are considered to be useful in diagnosing esophageal achalasia in Japan. According to the Japanese guidelines, ${ }^{2}$ there are 5 criteria to diagnose patients with esophageal achalasia by using esophagography. The availability of timed barium esophagram, which is an indicator of decreased clearance from the esophagus to the stomach, by measuring the height and width of residual barium in the esophagus at 1,2 , and 5 minutes after the administration of a certain amount of low-density barium, has recently been reported and is receiving increasing attention from the medical community for its use in diagnosing esophageal achalasia. ${ }^{4}$

Although sleeve sensors have been primarily used in esophageal manometry to diagnose esophageal achalasia, accurate diagnosis was difficult in some patients. This led to the development of HREM, which can evaluate the distance from the upper esophageal sphincter to the LES in a way that allows for a detailed assessment of the movement in the entire esophagus. HREM has been established as the gold standard test for examining patients with esophageal acha- 
lasia. Unfortunately, it has not yet been widely adopted in Japan, except in certain special institutions, because of the cost of the device.

Howard et $\mathrm{al}^{5}$ compared the diagnostic efficacy of conventional esophageal manometry and esophagography in 33 patients with achalasia, and found a significant difference between the procedures. Specifically, achalasia was diagnosed in $94.0 \%(\mathrm{n}=31)$ of the patients with esophageal manometry alone, and in $45.5 \%$ ( $\mathrm{n}$ $=15$ ) with esophagography alone. In another study, the authors reported that esophageal manometry was superior to esophagography in diagnosing achalasia. In 38 patients in whom achalasia was diagnosed using esophageal manometry, achalasia was diagnosed in $22(58.0 \%)$ patients with esophagography alone and could not be diagnosed in $16(42.0 \%)$ patients with esophagography alone. ${ }^{6}$ Although conventional esophageal manometry was used in these 2 previous studies, to our knowledge, no report exists on the comparative diagnostic efficacy of esophagography and HREM, which is the gold standard for assessing esophageal motility disorders. In our study, esophagography did not allow for a definitive diagnosis in approximately $20 \%$ of patients who were diagnosed as having esophageal achalasia based on the Chicago classification by using HREM.

These data indicate that there are limitations in the use of esophagography, by which esophageal achalasia is diagnosed based on a dilatated esophagus. We believe that HREM should be proactively used in patients presenting with a chief complaint of difficulty in swallowing, which is challenging to diagnose using EGD and esophagography.

In our study, the 10 cases in which achalasia could not be diagnosed using esophagography alone may have been pre-achalasia diffuse esophageal spasm (DES) or vigorous achalasia. A case of progression from DES to achalasia was first reported by Kramer et $\mathrm{al}^{7}$ and subsequently documented in 6 patients by Vantrappen et al. ${ }^{8}$ This result suggested that esophageal spasm and achalasia belong to the same spectrum of motor disorders. Therefore, patients with spasm and significant dysphagia merit further follow-up. Vigorous achalasia, first reported by Sanderson et $\mathrm{al}^{9}$ in 1967, is an esophageal motility disorder characterized by both non-peristaltic esophageal simultaneous contractions, as seen in DES, and the incomplete relaxation of the LES, as seen in achalasia. The transition from DES to vigorous achalasia ${ }^{10}$ and that from vigorous achalasia to classic achalasia ${ }^{11}$ has been previously reported. In vigorous achalasia, esophageal barium imaging shows a dilatated esophagus and abnormal spasm. However, as vigorous achalasia is relatively less common than classic achalasia in the clinical setting, future studies and data are needed for an accurate diagnosis.
Additionally, we examined epigastric digestive symptoms caused by esophageal achalasia and inferred that most patients with type I or type II achalasia had symptoms of dysphagia, which may have been caused by a dilatated esophagus. However, in a previous study, the common chief complaint of patients with type III achalasia was chest pain. ${ }^{12}$ Chest pain in these patients was thought to be primarily caused by the strong contraction of the esophagus, and that these patients had significantly higher esophageal pressure during swallowing than those with type I or II. ${ }^{13-16}$ In our study, we found that $50 \%$ (3 of 6 ) of patients with corkscrew-like findings on esophagography were classified as having type III achalasia. In addition, $75 \%$ of patients with type III achalasia had chest pain, and this result is consistent with those of previous studies.

We also examined the patients' epigastric digestive symptoms and health-related QOL. Patients with chest pain have been shown to have significantly lower health-related QOL than those without chest pain. ${ }^{17}$ Patients with type III esophageal achalasia who commonly reported chest pain had significantly lower physical QOL than those with type I and II esophageal achalasia. This may be due to a QOL reduction in patients, triggered by upper abdominal symptoms, specifically chest pain. However, according to another report, there were no statistically significant differences among patients presenting with type I, II, and III esophageal achalasia with respect to QOL. ${ }^{18}$ Thus, further studies with a larger subject population are needed.

We acknowledge that our study has certain limitations. First, as this was a single-institution retrospective study, the characteristics of our study subjects may not be representative of the general population. Second, we examined a small population of subjects. These factors may have influenced the study results. In addition, patients with type III achalasia were older than other types in this study. This result may have affected lower PCS in Type III. However, there have been no previous studies comparing age and QOL in patients with achalasia. Therefore, further studies seem to be needed to evaluate these clinical characteristics in patients with achalasia.

Diagnosing esophageal achalasia by using esophagography alone based on the type and degree of esophageal dilatation has limitations. Therefore, HREM should be used in patients presenting with dysphagia as the chief complaint and in whom achalasia cannot be diagnosed using EGD or esophagography.

\section{Financial support: None.}

\section{Conflicts of interest: None.}


Author contributions: Takahisa Yamasaki and Toshihiko Tomita contributed to the concept and design of the study; Takahisa Yamasaki, Toshihiko Tomita, and Sumire Mori were involved in data acquisition and analysis; Takahisa Yamasaki, Toshihiko Tomita, Sumire Mori, Mayu Takimoto, Akio Tamura, Ken Hara, Takashi Kondo, Tomoaki Kono, Katsuyuki Tozawa, Yoshio Ohda, Tadayuki Oshima, Hirokazu Fukui, Jiro Watari, and Hiroto Miwa contributed to data interpretation, critically reviewed the manuscript for important intellectual contents, and approved final version of the manuscript; Tadayuki Oshima provided statistifical design; and Hiroto Miwa coordinated acquisition of data and trial supervision.

\section{References}

1. Sadowski DC, Ackah F, Jiang B, Svenson LW. Achalasia: incidence, prevalence and survival. A population-based study. Neurogastroenterol Motil 2010;22:e256-e261.

2. Descriptive rules for achalasia of the esophagus. The Japan Society of Esophageal Diseases and Kanehara Co, Ltd, 2012. Available from URL: http://www.esophagus.jp/private/mypage/files/esophageal achalasia final.pdf (accessed 11 Jun 2018).

3. Bredenoord AJ, Fox M, Kahrilas PJ, et al. Chicago classification criteria of esophageal motility disorders defined in high resolution esophageal pressure topography. Neurogastroenterol Motil 2012;24(suppl 1):57-65.

4. Rohof WO, Lei A, Boeckxstaens GE. Esophageal stasis on a timed barium esophagogram predicts recurrent symptoms in patients with longstanding achalasia. Am J Gastroenterol 2013;108:49-55.

5. Howard PJ, Maher L, Pryde A, Cameon EW, Heading RC. Five year prospective study of the incidence, clinical features, and diagnosis of achalasia in Edinburgh. Gut 1992;33:1011-1015.

6. El-Takli I, O’Brien P, Paterson WG. Clinical diagnosis of achalasia: how reliable is the barium x-ray? Can J Gastroenterol 2006;20:335-337.

7. Kramer P, Harris LD, Donaldson RM Jr. Transition from symptomatic diffuse spasm to cardiospasm. Gut 1967;8:115-119.

8. Vantrappen G, Janssens J, Hellemans J, Coremans G. Achalasia, diffuse esophageal spasm, and related motility disorders. Gastroenterology 1979;76:450-457.

9. Sanderson DR, Ellis FH Jr, Schlegel JF, Olsen AM. Syndrome of vigorous achalasia: clinical and physiologic observations. Dis Chest 1967;52:508-517.

10. Millan MS, Bourdages R, Beck IT, DaCosta LR. Transition from diffuse esophageal spasm to achalasia. J Clin Gastroenterol 1979;1:107-117.

11. Bondi JL, Godwin DH, Garrett JM. "Vigorous" achalasia. Its clinical interpretation and significance. Am J Gastroenterol 1972;58:145-155.

12. Pratap N, Kalapala R, Darisetty S, et al. Achalasia cardia subtyping by high-resolution manometry predicts the therapeutic outcome of pneumatic balloon dilatation. J Neurogastroenterol Motil 2011;17:48-53.

13. Rohof WO, Salvador R, Annese V, et al. Outcomes of treatment for achalasia depend on manometric subtype. Gastroenterology 2013;144:718725 .

14. Pandolfino JE, Kwiatek MA, Nealis T, Bulsiewicz W, Post J, Kahrilas PJ. Achalasia: a new clinically relevant classification by high-resolution manometry. Gastroenterology 2008;135:1526-1533.

15. Boeckxstaens GE, Annese V, des Varannes SB, et al. Pneumatic dilation versus laparoscopic Heller's myotomy for idiopathic achalasia. N Engl J Med 2011;364:1807-1816.

16. Zaninotto G, Costantini M, Rizzetto C, et al. Four hundred laparoscopic myotomies for esophageal achalasia: a single centre experience. Ann Surg 2008;248:986-993.

17. Ortiz-Garrido O, Ortiz-Olvera NX, González-Martínez M, et al. [Clinical assessment and health-related quality of life in patients with noncardiac chest pain.] Rev Gastroenterol Mex 2015;80:121-129. [Spanish]

18. Ross D, Richter J, Velanovich V. Health-related quality of life and physiological measurements in achalasia. Dis Esophagus 2017;30:1-5. 